\title{
Trends in Reproductive Health indicators in Ethiopia: 2000-2014
}

\author{
Mulu Abraha Woldegiorgis*1 ${ }^{*}$, Jahar L. Bhowmik ${ }^{1}$, Janet E. Hiller ${ }^{2}$, Wubegzier Mekonnen ${ }^{3}$ \\ ${ }^{1}$ Statistics, Data Science and Epidemiology, Swinburne University of Technology, Melbourne, Australia \\ ${ }^{2}$ Health Sciences, Swinburne University of Technology, Melbourne, Australia \\ ${ }^{3}$ Public Health, Addis Ababa University, Addis Ababa, Ethiopia
}

Received: September 15, 2016

DOI: $10.5430 /$ ijh.v3n1p17
Accepted: November 20, 2016 Online Published: December 13, 2016

URL: http://dx.doi.org/10.5430/ijh.v3n1p17

\begin{abstract}
Introduction: The Millennium Development Goals include a focus on Reproductive Health (RH) indicators which are of substantial importance in low and middle income countries. However, little has been reported of the time-trend in these indicators in Ethiopia. This study aims to describe trends in RH indicators in Ethiopia from 2000-2014.

Methods: This study is a time-trend analysis of RH indicators. We used 15-years of RH indicator data sourced mainly from Federal Ministry of Health and Demographic and Health Survey. Maternal mortality ratio, antenatal care coverage, skilled birth attendance coverage, postnatal care coverage, contraceptive prevalence rate and total fertility rate were selected and analysed using numerical and graphic summaries. We used coefficient of determination $\left(\mathrm{R}^{2}\right)$ for the Federal Ministry of Health facility based data to examine the variation in annual progress explained by the regression model. Confidence intervals were used in the Demographic and Health Survey data to indicate the period when significant difference was observed. Trends of indicators from both data sources were also compared.

Results: During the 15 -years study period, antenatal care coverage increased from $29 \%$ to $98 \%\left(\mathrm{R}^{2}=95 \%\right)$. It increased more rapidly than skilled birth attendance which remained low at $41 \%$ in 2014. Postnatal care coverage increased from 5\% to $64 \%$ $\left(\mathrm{R}^{2}=96 \%\right)$. Modern contraceptive prevalence rate among the married women improved from 6.3\% (5.8\%, 6.8\%) to 40\% (38.7\%, $41.3 \%$ ) within the 15-year period. Maternal mortality ratio decreased from 990 in 2000 to 420 in 2013. The total fertility rate decreased from 5.5 to 4.1 children per woman. With lowest performance in 2000, Ethiopia demonstrated better improvement compared to selected sub-Saharan African countries. Variations in the trends were observed when data from both sources were compared.

Conclusions: RH service coverages have shown remarkable improvement while maternal mortality ratio, although reduced, remained high. There was a significant gap in the continuum of maternal health services.
\end{abstract}

Key Words: Trend, Antenatal care, Skilled birth attendance, Postnatal care, Family planning, Fertility, Maternal mortality, Ethiopia

\section{INTRODUCTION}

Reproductive health (RH) is considered as a state of complete physical, mental and social well-being and not merely the absence of disease or infirmity, in all matters relating to the reproductive system and to its functions and processes. ${ }^{[1]}$ It is central to what it means to be human and is of critical importance at an individual, societal and global level. ${ }^{[2,3]}$ Africa's current challenges in effectively realising RH for its

*Correspondence: Mulu Abraha Woldegiorgis; Email: mwoldegiorgis@ swin.edu.au; Address: John Street, Hawthorn, Victoria 3122, Australia. 
people include unmet needs in access to contraception, and high levels of maternal mortality and morbidity from lack of access to obstetric care. ${ }^{[4]}$ In 2000 , the average risk of dying during pregnancy or childbirth in the developing world was 450 per 100,000 live births. ${ }^{[5]}$ In 2013, sub-Saharan Africa (SSA) was the only region worldwide with a very high maternal mortality ratio (MMR), accounting for $62 \%(179,000)$ of global maternal deaths. ${ }^{[6,7]}$

The global health view is that, many health problems in pregnant women can be prevented, detected and treated during antenatal care (ANC) visits. For this purpose, it is recommended that a pregnant woman should have at least four ANC visits. ${ }^{[8,9]}$ Accordingly, efforts to monitor progress in coverage of ANC should focus on quantifiable variables such as the number and timing of visits and the characteristics of users and non-users of ANC. ${ }^{[10]}$ Having an adequate number of ANC visits and receiving quality care during those visits can ensure that women are well prepared for the childbirth. ${ }^{[11,12]}$ However, many of the interventions known to save the lives of women and their newborns also depend upon the presence of a skilled birth attendants. ${ }^{[13]}$

Total fertility rate (TFR) is closely associated with Contraceptive Prevalence Rate (CPR) and other indicators of RH such as MMR. TFR is a useful indicator of population momentum and a good proxy measure for the success or failure of family planning (FP) services. ${ }^{[14]}$ The most substantial benefits of contraceptive use for the health and survival of women and children stem from reductions in the number of pregnancies, especially those that are a greater-than-average risk to maternal, perinatal, and child survival. ${ }^{[15]}$

Ethiopia, the second most populous country in Africa, with a total population of over 90 million, has one of the poorest $\mathrm{RH}$ levels as indicated by high rates of maternal mortality. ${ }^{[16,17]}$ To address this problem, the country has had a significant expansion of health care facilities with more than 35,000 community health workers called health extension workers (HEW) mainly engaged in maternal and child health activities. ${ }^{[18,19]}$ By 2015, the country aimed to meet the millennium maternal mortality goal of 350 maternal deaths per 100,000 live births. ${ }^{[20]}$ The target set by the Federal Ministry of Health (FMOH) of Ethiopia documented in the Health Sector Development Program (HSDP) IV was even to decrease further to 267 per 100,000 live births by 2015. ${ }^{\text {[21] }}$

Annual progress in the utilization of RH services and improvements in $\mathrm{RH}$ status have been aggregated and reported in Ethiopia over the past two decades. ${ }^{[21-26]}$ However, time trends in the progress of the main RH indicators have not yet been systematically investigated. Therefore, the aim of this study is to describe the trends in $\mathrm{RH}$ indicators in Ethiopia for the period 2000-2014.

\section{METHODS AND MATERIALS}

This study is a 15-years trend analysis of RH indicators using nationally representative data along with qualitative data and other international sources relevant to RH status development in Ethiopia.

\subsection{Indicator selection}

Among the $17 \mathrm{RH}$ indicators agreed by international agencies for monitoring the RH goals, ${ }^{[14]} \mathrm{MMR}$, ANC coverage, Skilled Birth Attendance (SBA) coverage, TFR and CPR are selected based on their relevance to policy and programming as well as data availability. Postnatal Care (PNC) coverage is also included as a sixth study indicator as it is critical to the health and survival of a mother and the new born. ${ }^{\text {[27] }}$

\subsection{Quantitative data sources}

The Health and Health Related Indicators (HHRI) data, from 2000 to 2014, of Ethiopia's Health Management Information System (HMIS) prepared by the FMOH as well as Ethiopia's 2000, 2005 and 2011 standard Demographic and Health Surveys (DHS) data, accessed with permission from the DHS program ICF INTERNATIONAL were the main data sources. DHS VI recode manual ${ }^{[28]}$ was used as a guide while analysing DHS data. We used a sampling weights using the guide to DHS statistics. ${ }^{[29]}$ Ethiopian 2007 and 2014 HMIS indicator definitions, ${ }^{[30,31]}$ and the WHO 2006 $\mathrm{RH}$ indicator guidelines ${ }^{[14]}$ were used to define the study indicators. With some expected variations due to methodological differences in data collection process, establishing eligible and data presentation, we compared RH trends between HMIS and DHS sources. Ethiopian RH trend data were summarized and compared with seven SSA countries whose DHS was conducted within a year of the Ethiopian's DHS rounds (2000, 2005 and 2011). The seven countries included in this aspect were Benin, Mail, Guinea, Namibia, Rwanda, Tanzania and Uganda.

SPSS Version 23 was used for data analysis. Both numeric and graphic methods were used to describe the indicator trends. Trend lines were used to describe the progress of $\mathrm{RH}$ indicators over time. In HMIS data, $\mathrm{R}^{2}$ was used to examine the variation of annual progress of the $\mathrm{RH}$ indicator that can be explained by the regression model, whereas in DHS data, a $95 \%$ confidence interval was used to determine the years when statistically significant changes were observed over the course of the analysis period. In the analysis of trends, comparisons were made between the two data sources. 


\subsection{Qualitative data}

The study also involved expert interview/consultation regarding irregular trends (sharp fall/increase), RH data and service quality issues of the HMIS as well as data discrepancy between HMIS and DHS sources. Twelve experts (6 M\&E and $6 \mathrm{RH}$ program experts) were selected based on one or more of the following criteria: Expertise at RH or M\&E; practical experience in the Ethiopian HMIS; involved at the different stages of DHS, worked at RH development partner organization; participated at health related special support (supervision) to pastoralist regions, involved at any performance monitoring/review process. Ethical approval was obtained from Swinburne University of Technology, Subcommittee (SHESC3) of Swinburne's Human Research Ethics Committee (SUHREC), reference number: SHR Project 2015/121. All participants were requested to read the consent and information statement forms before conducting the interview. We used email, telephone, in-person and self-administrated techniques as per the preference of the interviewees. The in-depth interview results were summarized and discussed along with the quantitative findings.

\section{RESULTS}

\subsection{Antenatal care (ANC)}

According to the HMIS data, the overall increase in ANC coverage during the 15 -year study period was $69 \%$. The proportion of expected pregnant mothers (estimated from the 2007 population and housing census) who received ANC increased from $29.1 \%$ in 2000 to $98.1 \%$ in 2014 . Overall, $67 \%$ of the expected pregnant mothers received ANC service during the entire period. The lowest ANC coverage was in $2003(27 \%$ ) with a declined from $34 \%$ in 2002 . In $2014,98 \%$ of the pregnant mothers in the country received the service. ANC coverage followed a linear trend with a 5\% average annual increase. As indicated in Figure 1, 95\% of the changes in ANC could be explained by the linear regression model.

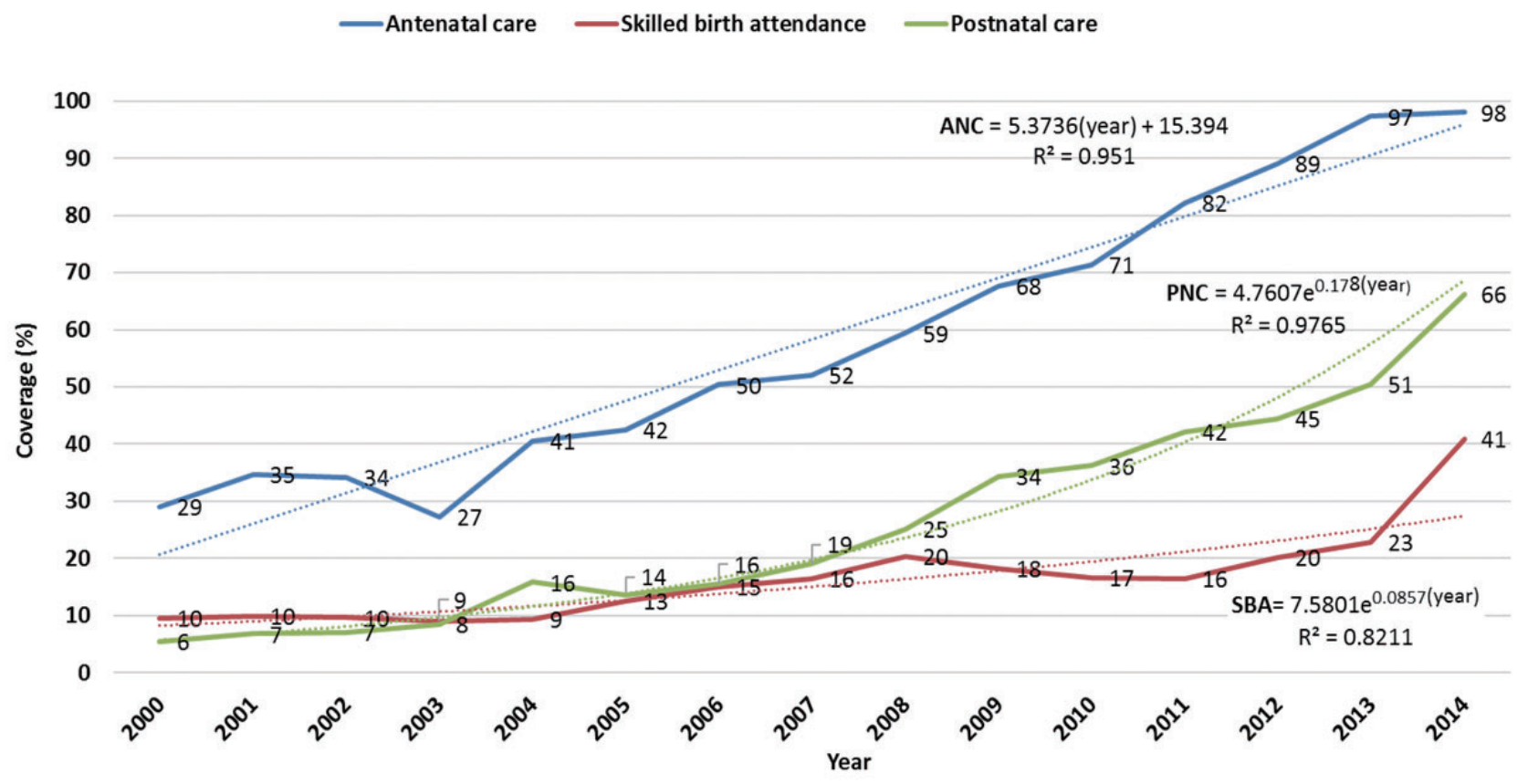

Figure 1. Trends of antenatal, skilled birth attendant and postnatal care coverages in Ethiopia from 2000-2014 Data source: Ethiopia Federal Ministry of Health of Ethiopia, Health and Health related indicator reports 2000-2014

Table 1 shows a comparison between DHS and HMIS indicator values for the years 2000, 2005 and 2011. Noticeable progresses were shown in both sources with significant difference between them for the same indicator. As HMIS progressed more rapidly than DHS, their difference in all indicators increased with time. While HMIS indicated an increase in ANC1+ by 53\%, a $16 \%$ increase was seen in DHS between 2000 and 2011.

Published by Sciedu Press

\subsection{Skilled birth attendance (SBA)}

Low coverage of SBA has been a challenge for many years. In 2000 , only $9.6 \%$ of pregnant women gave birth with the assistance of skilled health workers. According to HMIS, a $31.3 \%$ improvement was observed over the 15 -year period. The trend indicated an exponential increase with much higher progress during the last three years (2012-2014) than the previous 12 years (2000-2011). Highest change was observed in 
2014 which moved the coverage from $23 \%$ in 2013 to $41 \%$ (see Figure 1). However, it is still evident that more than half $(59 \%)$ of the delivering mothers were not being assisted by skilled health personnel (of which $8 \%$ were assisted by
HEWs). As shown in Table 1, difference can be seen between the two sources. DHS indicated low coverage in all rounds with significant but minor change between 2000 and 2011.

Table 1. Comparison of RH indicators by source (DHS Vs HMIS)

\begin{tabular}{lllllll}
\hline \multirow{2}{*}{ Year } & \multirow{2}{*}{ Source } & \multicolumn{5}{c}{ Indicator } \\
\cline { 3 - 7 } & & ANC 1+ & SBA & PNC 48 hrs & PNC 6 wks & CPR/CAR \\
\hline \multirow{2}{*}{$\mathbf{2 0 0 0}$} & HMIS & 29.1 & 9.6 & & 5.5 & 13 \\
& DHS (CI) & $27.3(26.3,28.3)$ & $6.2(5.7,6.7)$ & $7.8(7.2,8.4)$ & $10(9.4,10.7)$ & $6.3(5.8,6.8)$ \\
\multirow{2}{*}{$\mathbf{2 0 0 5}$} & HMIS & 42.5 & 12.5 & & 13.6 & 26 \\
& DHS (CI) & $28.4(27.4,29.4)$ & $7(6.4,7.6)$ & $4.6(4.1,5.1)$ & $5.9(5.4,6.5)$ & $13.7(13.2,14.6)$ \\
\multirow{2}{*}{$\mathbf{2 0 1 1}$} & HMIS & 82.2 & 16.4 & & 42.1 & 62 \\
& DHS (CI) & $42.9(41.9,43.9)$ & $11.8(11.1,12.5)$ & $6.7(6.0,7.5)$ & $8.3(7.5,9.2)$ & $27.3(26.4,28.2)$ \\
\hline
\end{tabular}

Note. ANC1+ refers one or more Antenatal care visit/s, SBA refers skilled birth attendance, PNC 48 hrs refers Postnatal care given within 48 hrs, PNC 6 wks refers Postnatal care coverage within 6 weeks of delivery. CPR/CAR refers CPR (Contraceptive prevalence rate) for DHS data and CAR (contraceptive acceptance rate) for HMIS data.

\subsection{Postnatal care (PNC)}

Similar to the trends observed in ANC and SBA, considerable improvement was observed in PNC coverage in 15-year period. At the beginning of the study period, PNC coverage was limited to only $5 \%$ of the expected deliveries which was $4 \%$ less than SBA coverage. Like SBA, this indicator also followed an exponential trend with $\mathrm{R}^{2}$ of $96 \%$, but had rapid progress. By the end of the study period (2014), PNC coverage of the country reached $64 \%$ while SBA remained at $41 \%$ (see Figure 1). This revealed that a significant proportion of mothers who delivered without the assistance of skilled health workers might have received PNC services. The comparison in trends between HMIS and DHS data shows lower coverage across all the DHS rounds. In 2011, PNC coverage of HMIS was five times higher than DHS. The trend in the proportion of mothers who received PNC service within the first 48 hours ( 2 days) was even much less.

\subsection{Family planning}

Contraceptive Acceptance Rate (CAR) among married women from HMIS source followed a linear trend and improved by an average of $4 \%$ each year with an overall improvement of 50\%, from $13 \%$ in 2000 to $63 \%$ in 2014 . While comparing between the two data sources, we acknowledge that some differences could be attributed to the slight difference in indicator definition. There was an improvement in both data sources during the 15-year period. As shown in Table 1, CAR of HMIS was nearly twice that of CPR of HMIS in all periods. CPR improved by $21 \%$, while CAR increased by $49 \%$ during the study period (see Table 1 ).

Currently married women who do not want any more children or want to wait at least two years before having another child, but who are not using contraception, are considered to have an unmet need for family planning (FP). The sum of unmet need and met need (currently using FP methods) constitute the total demand for FP. ${ }^{[32]}$ Unmet need for FP decreased from $35.8 \%(34.8,36.8)$, to $25.3 \%(24.5,26.1)$ between 2000 and 2011 whereas total demand increased from $43.8 \%(42.8,44.8)$ to $53.9 \%(53.9,54.9)$. Nearly $11 \%$ of unmet need for FP had been addressed within the 12-year period while the total demand for FP increased by $10 \%$.

\subsection{Total fertility rate (TFR)}

Results indicate that TFR decreased from $5.5(5.1,5.9)$ children per woman in 2000 to $4.8(4.5,5.1)$ in 2011. The HMIS does not include this indicator as it is not possible to compute TFR from health facility data.

\subsection{Maternal mortality ratio (MMR)}

An inter-agency group from the WHO, UNICEF, UNFPA, the World Bank and UN population division used a model to estimate the MMR for Ethiopia for the period 1990-2013.[22] According to this model, MMR decreased from 990 (580, $1700)$ per 100,000 live births in 2000 to $420(240,720)$ in 2013, a decrease of 570 per 100,000 live births. Results obtained from the DHS data indicated a decrease from 871 $(703,1039)$ in 2000 to $676(541,810)$ in 2011 (see Table 2).

An institutional maternal death rate is the proportion of maternal deaths from all deliveries attended in a given institution. It is defined as the death of a woman from conditions caused by pregnancy, which occurs from time of conception to six weeks postpartum, but not from incidental or accidental. ${ }^{[31]}$ This indicator is part of the HMIS indicators reported by the FMOH which is calculated as the number of maternal deaths in a health institution divided by the total number of deliveries in the health institution during a given time 
period, usually one year. Data were only available for the period 2010-2012. The data indicate that institutional maternal death rate was high in 2011 (331 per 100,000 deliveries) compared to 2010 and 2012 (see Table 3).

\section{Comparison of trends of $\mathrm{RH}$ indicators with selected coun- tries}

Table 4 shows maternal health and fertility indicators among the selected SSA countries. It can be seen that Ethiopia's maternal health service coverage across time was far below the other countries. Ethiopia's CPR for the years 2005 and 2011 show above the median and was the highest improve- ment next to Rwanda. The TFR for all selected countries decreased by 0.7 or more in 15 years except Tanzania.

\section{DisCUSSION}

This study focuses on Ethiopia's RH progress in comparison with the national and international initiatives/targets using quantitative data and experts' opinion. Trends in the overall $\mathrm{RH}$ and continuum of maternal health services (ANC, SBA and PNC) are assessed. The discrepancy between the two main data sources - the HMIS and the DHS - are discussed. On the basis of the obtained results, some suggestions are addressed.

Table 2. Maternal Mortality ratio per 100,000 live births in Ethiopia for the period 1990-2013 by Inter-agency estimates and Demography and Health Survey

\begin{tabular}{lllll}
\hline \multirow{2}{*}{ Year } & MMR per 100,000 live births (95\% CI) & \multicolumn{4}{l}{ MMR Per 100,000 live births, By DHS } \\
\cline { 3 - 5 } & By interagency group (estimate) & & Size of survey sample & Period included \\
\hline 1990 & $1,400(790,2,300)$ & & & \\
1995 & $1,200(710,2,100)$ & $871(703,1,039)$ & 15,367 & 1993,2000 \\
2000 & $990(580,1,700)$ & $673(548,799)$ & 14,070 & 1998,2005 \\
2005 & $740(440,1,300)$ & $676(541,810)$ & 16,515 & 2004,2011 \\
2011 & & & & \\
2013 & $420(240,720)$ & & \\
\hline
\end{tabular}

Table 3. Intuitional maternal death in Ethiopia for the period 2010-2012

\begin{tabular}{llll}
\hline \multirow{2}{*}{ Years } & \multirow{2}{*}{ Number of deliveries } & \multicolumn{2}{c}{ Institutional Maternal Deaths } \\
\cline { 3 - 4 } & & Number & Proportion (Maternal death/ total deliveries * 100,000) \\
\hline 2010 & 497,328 & 855 & 171 \\
2011 & 485,809 & 1,597 & 331 \\
2012 & 615,256 & 1,033 & 169 \\
\hline
\end{tabular}

\subsection{Trends and continuity of maternal health services}

\subsubsection{Antenatal care and skilled birth attendance cover-} age

The time trend analysis of HMIS data indicates that Ethiopia's ANC coverage improved more rapidly (3.5 times higher) than SBA. SBA remained low throughout the study period but showed sharp (23\%) improvement in 2014. M\&E experts interviewed about the implication of the sharp increase explained this major shift as due to service expansion, special attention by the Ethiopian government and high involvement of RH partners to strengthen the quality and equity of maternal and child health service. A new indicator called "proportion of kebeles (smallest administration unit) declared home delivery free" included at HMIS in 2014 for routine monitoring ${ }^{[33]}$ can be seen as readiness to improve SBA services. This indicator has been introduced to further strengthen implementation of community level integrated maternal and child health services and to create positive

Published by Sciedu Press competition among the neighbouring kebeles.

DHS also showed higher increase in ANC. For example, the 2014 mini-DHS data indicated that ANC was more than twice of SBA. This finding is consistent with the 1990-2001 SSA trend study conducted by the WHO in which the levels for ANC use were consistently higher than that of SBA. ${ }^{[10]}$ Such difference might also be attributed to the fact that, unlike SBA, ANC service is being provided and reported by the community HEWs at health post/lowest primary health unit. $^{[33,34]}$ This is also evidenced by M\&E experts that the HEWs at health posts have had high impact for improving ANC while SBA service is limited to health centres and hospitals. However, program experts argued that this is the weakness of the health system in implementing the primary health care unit because HEWs are expected to improve SBA by ensuring all pregnant mothers to receive skilled delivery service through referral linkage within the primary health care unit. Another study explained that ANC is less effec- 
tive in SSA in getting women to use skilled attendance at delivery partly due to the fact that indicators on use of ANC services provide no information on the content or quality of the services. ${ }^{[10]}$ A further analysis of the most recent DHS surveys conducted in 44 developing countries between 2000 and 2010 on place of birth for all children born five years preceding the surveys indicated a shift in SSA region in favour of home delivery ${ }^{[35]}$ which is one indication of poor service quality. To improve SBA, a study in Cameroon suggested a model of optimal ANC which is at least four visits, the first in the first trimester, the last in the third trimester, and at least one visit from a skilled health provider. ${ }^{[36]}$ Our study suggests that indicators focusing on quality and continuity of ANC services need to be added as part of the routine health system monitoring.

Table 4. RH coverage of some Sub Saharan African countries conducted within a year of the three Ethiopia's DHS periods

\begin{tabular}{|c|c|c|c|c|c|c|c|c|c|c|c|c|}
\hline \multirow[b]{2}{*}{ Country } & \multicolumn{4}{|c|}{$1999 / 2000 / 2001$} & \multicolumn{4}{|c|}{$2004 / 2005 / 2006$} & \multicolumn{4}{|c|}{$2010 / 2011 / 2012$} \\
\hline & ANC(4+) & SBA & $\begin{array}{l}\text { Modern } \\
\text { CPR }\end{array}$ & TFR & ANC(4+) & SBA & $\begin{array}{l}\text { Modern } \\
\text { CPR }\end{array}$ & TFR & ANC(4+) & SBA & $\begin{array}{l}\text { Modern } \\
\text { CPR }\end{array}$ & TFR \\
\hline Benin & 63.2 & 67.8 & 7.2 & 5.6 & 61.5 & 76.4 & 6.1 & 5.7 & 61.1 & 82.1 & 7.9 & 4.9 \\
\hline CI & $61.6,64.8$ & $66.3,69.3$ & $6.4,8.0$ & $5.0,6.2$ & $60.6,62.4$ & $75.6,77.2$ & $5.7,6.5$ & $5.4,6.0$ & $60.1,62.1$ & $81.3,82.9$ & $7.4,8.4$ & $4.6,5.2$ \\
\hline Ethiopia & 10.6 & 6.2 & 6.3 & 5.5 & 12.3 & 7.0 & 13.7 & 5.4 & 19.1 & 11.8 & 27.3 & 4.8 \\
\hline CI & $9.9,11.3$ & $5.7,6.7$ & $5.8,6.8$ & $5.1,5.9$ & $11.5,13.1$ & $6.4,7.6$ & $13.2,14.6$ & $5.0,5.8$ & $18.2,20.0$ & $11.1,12.5$ & $26.4,28.2$ & $4.5,5.1$ \\
\hline Guinea & 51.5 & 9.8 & 4.2 & 5.5 & 51.4 & 30.5 & 5.7 & 5.7 & 56.9 & 40.3 & 4.6 & 5.1 \\
\hline CI & 49.9, 53.1 & $8.9,20.7$ & $3.7,4.7$ & $5.0,6.0$ & $49.9,52.9$ & 29.1, 31.9 & $5.1,6.3$ & $5.2,6.2$ & $55.5,58.3$ & $38.9,41.7$ & $4.1,5.1$ & $4.6,5.6$ \\
\hline CI & $30.9,32.9$ & $23.2,25.0$ & $6.5,7.5$ & $6.4,7.2$ & $35.3,37.3$ & 28.1, 29.9 & $6.5,7.3$ & $6.2,7.0$ & $40.4,42.8$ & $39.2,41.6$ & $9.3,10.5$ & $5.6,6.6$ \\
\hline Rwanda & 10.6 & 25.7 & 5.7 & 5.8 & 13.4 & 29.6 & 10.3 & 6.1 & 35.5 & 71.9 & 45.1 & 4.6 \\
\hline CI & $9.8,11.4$ & $24.5,26.9$ & $5.1,6.3$ & $5.4,6.2$ & $12.5,13.4$ & $28.4,30.8$ & $9.5,11.1$ & $5.7,6.5$ & $24.4,36.6$ & $70.8,73.0$ & $43.9,46.3$ & $4.2,5.0$ \\
\hline Tanzania & 71.1 & 38.5 & 16.9 & 5.6 & 61.7 & 45.6 & 20 & 5.7 & 43.2 & 51.5 & 27.4 & 5.4 \\
\hline CI & 69,73 & $36.1,40.9$ & $15.5,18.3$ & $4.8,6,4$ & $60.4,63.0$ & $44.3,46.9$ & $19.1,20.9$ & $5.3,6.1$ & $41.9,44,5$ & $50.2,2.8$ & $26.3,28.5$ & $5.0,5.8$ \\
\hline Uganda & 42.9 & 19.8 & 18.2 & 6.9 & 47.7 & 45.2 & 17.9 & 6.7 & 48.5 & 60.1 & 26.0 & 6.2 \\
\hline CI & $41.4,44.4$ & 18.6, 21.0 & $17.1,19.3$ & $6.3,7.5$ & $46.3,49.1$ & $43.8,46$ & $16.9,18.9$ & $6.2,7.2$ & $47.1,49.9$ & $58.7,61.5$ & $24.8,27.2$ & $5.7,6.7$ \\
\hline
\end{tabular}

Note. DHS period of Benin (2001, 2006 and 2011/12), Ethiopia (2000, 2005 and 2011), Guinea (1999, 2005 and 2012), Mali (2001, 2006 and 2012/13), Rwanda (200/01, 2006 and 2011), Tanzania (1999, 2004/05 and 2010) and Uganda (2001, 2006 and 2012/13).

\subsubsection{Postnatal care coverage}

According to HMIS data, the annual incremental improvement in PNC was better than SBA. This indicates increasing trend of mothers giving birth at home and appeared at health facilities for PNC services. On the other hand, since PNC is one of the services provided by HEW at a health post level (unlike SBA), this could increase the coverage. Regarding the timing of PNC, Ethiopia's RH programs encourage a visit within the first 7 days after delivery, mainly the first 2 days. ${ }^{[33]}$ In this case, DHS data showed that small proportion (11.8\%) of women received PNC service in the first two days after birth. There is a huge difference in PNC coverage between HMIS and DHS sources and experts' opinions are summarized as follows. In some cases, there are issues with regard to women who delivered at home and visited health facility within 6 weeks of delivery. Firstly, even though PNC service was provided for the mother as well, some women might not realize it because of lack of proper advice by health workers. Secondly, some might not value any service given to them as their intended purpose for vising the health facility was for their infants. Thirdly, the mother might be recorded as having received PNC services by default just due to the timing of her appearance to health facility even though only the child had practically received the service (e.g. vaccination). These cases might only be reported in HMIS, but not in DHS. A proposed suggestion by MEASURE Evaluation states that PNC statistics should make explicit whether care was provided principally for the mother or baby or both mother and baby. ${ }^{[37]}$ This would help to minimize the discrepancy between them in line with improving other data quality problems.

\subsubsection{Contraceptive prevalence/acceptance rate}

Ethiopia's CPR goal for 2015 was to reach $65 \% .{ }^{[38]}$ A $42 \%$ coverage in CAR was achieved in 2011 according to HMIS while DHS data showed 27.3\%. This huge difference between both sources might partly be due to the difference in indicator definition. CAR considers repeat and new users who appear to the health facility and receive the service at least once during the fiscal year whereas DHS considers users at the time of the survey. Key informants also added that there is likelihood of double /multiple counting in CAR as some health workers do not understand the concepts of "new" and "repeat" acceptors.

\subsubsection{Total fertility rate}

The $\mathrm{WHO}^{[14]}$ described that TFR is an important indicator as it closely associated with CPR and MMR. It is a useful indicator of population growth momentum and a good proxy measure for the success (or failure) of family planning service. ${ }^{[14]}$ Ethiopia's TFR target for 2015 was 4.0 children per woman. ${ }^{[21]}$ In 2014 , the TFR dropped to 4.1 with a total reduction of 1.4 children per women in 15 years. This achievement could be due to addressing unmet needs for FP 
and increasing access to contraceptive services.

\subsubsection{Maternal mortality ratio}

The inter-agency data indicated a steady decrease between 2000 and 2013 but with overlapping confidence intervals. Significant change was obtained in 2013 compared to 1990. DHS data also indicated a reduction in MMR but with overlapping confidence intervals across the three DHS rounds. Although improvement in the RH indicators can be seen over the 15 years period, MMR remained higher than the MDG target ${ }^{[20]}$ of $350 \mathrm{MMR}$ per 100,000 live births, as well as the Ethiopia's Growth and Transformation plan ${ }^{[39]}$ and HSDP IV $^{[21]}$ targets of $267 \mathrm{MMR}$ by 2015. The high institutional maternal death (see Table 3) also indicated high maternal deaths even after mothers arrived at health facilities. High institutional maternal death might result in decreasing demand for assisted/institutional delivery service.

\subsection{Data discrepancy between HMIS and DHS}

As described above, differences in coverages of ANC, SBA, PNC and contraceptive use were observed between DHS and HMIS sources. It is noticed that the DHS indicator values were consistently lower than that of HMIS. This difference was also noted in the FMOH annual HSDP IV performance report of 2014. This report suggested not to compare DHS with HMIS given the methodological differences as a reason. ${ }^{[19]}$ However, as both sources are official, researchers and program implementer organizations may wish to use both sources simultaneously. The health sector development programs, HSDP I ${ }^{[40]}$ and HSDP II $^{[41]}$ evaluation reports of FMOH also simultaneously used DHS and HMIS sources for the same indicators in a similar report. The HMIS data has been used especially for planning purpose as data are available on a yearly basis. The HSDP IV took baseline for SBA from HMIS data, ${ }^{[21]}$ whereas its performance evaluation report data were taken from DHS. ${ }^{[19]}$

In the interview, RH experts were asked to share their thoughts about the preferred data source by the governmental and/or non-governmental organizations as well as researcher institutions during planning and/or evaluation of $\mathrm{RH}$ program. Most experts preferred DHS by mentioning a variety of reasons: DHS data is more reliable as it has standardized tool; it follows scientific methodology and involves different stakeholders at different levels of the survey; the data collection, analysis and data quality check is done by well-trained experts. Moreover, plan/evaluation of a program using DHS data is more acceptable to any organization. A program expert also added; "Government uses the HMIS data sources whereas most non-government organizations use the DHS sources". On the other hand, an M\&E expert said "HMIS data is more reliable as it represents all health facilities in

Published by Sciedu Press the country even though it has data quality issues whereas DHS data comes from very limited sample of the population". An expert from RH partner organization also responded that HMIS data are more practically being used despite their data quality issues. Experts were also consulted about the possible ways of minimizing the discrepancy between the two sources. They all agreed that the discrepancy is high and unacceptable and suggested that HMIS should focus on quality of the RH services and indicators. It was also suggested that the FMOH should actively involve at the Ethiopian DHS 2016 by assigning appropriate experts during the design, data analysis and dissemination stages of the survey.

\section{CONCLUSION AND RECOMMENDATIONS}

The overall RH service coverage has improved during the 15 -year study period. ANC and PNC coverage showed noticeable improvement whereas the low ANC (+4) coverage, early PNC service, and SBA coverage need to be improved as they are key indicators for further improvement in maternal mortality. The health system need to assess the huge trend gap in the continuum of maternal health services, especially the low utilization of SBA service despite high ANC coverage. Tracking and improving the proportion of pregnant mothers with ANC4+ visits may improve the proportion of skilled deliveries as indicated in other SSA countries. Efforts need to be increased in reducing MMR as it is found to be higher than the MDG and HSDP IV targets. Institutional maternal death also pointed the need of improving quality of health service.

While this is a significant achievement over the 15 -year period, more research is needed to investigate the quality of the services and data. The wider gap in RH indicators between DHS and HMIS needs to be recognized and managed appropriately as both are key sources of information for many stakeholders during planning, evaluation as well as decision/action of RH in the country. With the current practice, the authors suggest that DHS could be more reliable source for researchers than HMIS data. This is due to the fact that DHS has better tool, data management and data quality ensuring techniques. Moreover, the individual level data of the DHS would be more informative for researchers and implementers than the aggregated HMIS data. However, as the HMIS has monthly and annual data, program implementers and evaluators might need to use HMIS for routine monitoring and evaluation. Our suggestion here is that; data analysts should be careful not to use both sources interchangeably for the same indicator as it could lead to a wrong conclusion especially while attributing changes to an intervention. Our suggestion to the policy makers is to place every possible effort for improving data credibility of the HMIS. 


\section{ACKNOWLEDGements}

Data for this study were extracted from health and health related indicators of Ethiopia 2000-2014 accessed from Ministry of Health (Ethiopia) website. The principal investigator is authorized by DHS program to access and use individual record DHS data of all SSA countries. Inter-Agency estimated data of MMR 1990-2013 were also used. We extend our acknowledgement to the owners and authors of these

\section{REFERENCES}

[1] UNFPA. Programme of Action of the International Conference on Population Development 20th Anniversary Edition. Available from: http://bit.1y/1Q8HrDd 2014.

[2] Ford J, Nassar N, Sullivan EA, et al. Reproductive health indicators Australia 2002. AIHW Cat No. PER 20. Canberra: AIHW NPSU. 2003.

[3] Ford J, Nassar N, Sullivan E. Reproductive Health Indicators, Australia, 2002. Australian Institute of Health and Welfare: Canberra 2003.

[4] Charles N. Strengthening the protection of sexual and reproductive health and rights in the African region through human rights. South Africa: Pretoria University Law Press; 2014.

[5] United Nations. The Millennium Development Goals Report 2005. New York. 2005.

[6] WHO, et al. Trends in maternal mortality 1990-2013 Estimates by WHO, UNICEF, UNFPA, The World Bank and the United Nations Population Division. 2014: Geneva, Switzerland.

[7] Human reproduction program, W., Strategies toward ending preventable maternal mortality (EPMM). 2015: Geneva, Switzerland.

[8] WHO, WHO programme to map best reproductive health practices, WHO Antnatal Care randomized trial: Manual for the implementation of the new Model. Geneva. 2002.

[9] United Nations. The Millennium Development Goals Report 2009. New York. 2009

[10] Abou-Zahr CLIW, Tessa M. Antenatal care in Developing countries: Promises, Achievmenets and missed opprtunities: an analysis of trends, levels and differentials, 1990-2001. 2003.

[11] Wenjuan W, Shanxiao W, Alfredo F. Levels and Trends in the use of Maternal Health Service in Developing countries, DHS Comparative Reports No. 26. Calverton, Maryland, USA. 2011.

[12] Central staticstics agency, I.I., Ethiopia Demography and Health Survey 2011. 2012

[13] Koblinsky M, et al. Issues in programming for safe motherhood. Arlington, Virginia: MotherCare, John-Snow, Inc. 2000; 38: 43.

[14] WHO RHR. Reproductive health indicators : guidelines for their generation, interpretation and analysis for global monitoring, R.H.a Research, Editor. 2006, World Health Organization, Stylus Pub Llc, C/O Books Intl, Po Box 605, Herndon, VA, 20172: Geneva, Switzerland. p.63.

[15] Cleland J, Conde-Agudelo A, Peterson H, et al. Contraception and health. The Lancet. 2012; 380(9837): 149-56. https://doi .org/ 10.1016/S0140-6736(12)60609-6

[16] Federal Ministry of Health, Health Sector Development Program II 2002/ 03 - 2004/ 05. 2002 Federal Ministry of Health: Addis Ababa, Ethiopia. data and reports. Our gratitude also goes to $\mathrm{RH}$ and M\&E experts for providing detail thoughts. We also thank Associate Professor Timothy Moore from Swinburne University for his editing help. Finally, we extend our gratitude to Swinburne university of Technology for all the support.

\section{CONFlicts OF InTEREST Disclosure}

The authors declare that there is no conflict of interest associated with this study.
[17] World Bank. Data-Sub Saharan Africa countries (Developing only). Available from: http://data.worldbank.org/region/ SSA 2015.

[18] Federal Ministry of Health, Ethiopia Health and Health Related Indicators 2013/2014 Version 1. 2014, Federal Ministry of Health: Addis Ababa, Ethiopia.

[19] Federal Ministry of Health, Health Sector Development Program annual perfomance Report o EFY 2006 (2013/2014) Version 1. 2014 Federal Ministry of Health,: Addis Ababa, Ethiopia.

[20] World Health Organization. Countdown to 2015, Ethiopian Health data - 2014 profile, count down to 2015. 2014.

[21] Federal Ministry of Health, Ethiopia Health Sector Development Program IV 2010/11-2014/15. 2010 Federal Ministry of Health,: Addis Ababa, Ethiopia.

[22] WHO, et al. Ethiopia Maternal Mortality 1990-2013. 2014.

[23] Worku AG, Tessema GA, Zeleke AA. Trends of Modern Contraceptive Use among Young Married Women Based on the 2000, 2005, and 2011 Ethiopian Demographic and Health Surveys: A Multivariate Decomposition Analysis. PLoS ONE. 2015; 10(1): 114. PMid: 25635389. https://doi.org/10.1371/journal.po ne. 0116525

[24] Blake Z, Pav G. Trends in Demographic and Reproductive Health Indicators in Ethiopia, Data from the 2000, 2005, and 2011 Demographic and Health Surveys 2013.

[25] Ayele W, et al. Trends and Determinants of unmet need forfamily planning, and progrma options, Ethiopia, Further analysis of Ethiopian DHS, 2000, 2005 and 2011. Further Analysis of the EDHS, 2013.

[26] Federal Ministry of Health, Federal Demogratic Republic of Ethiopia HSDP IV annual performance report. 2011: Addis Ababa, Ethiopia.

[27] Charlotte W, Lalla T, Pyande M. Postnatal care. [cited 2015 Jan 10]; Available from: http://www . who.int/pmnch/media/publicat ions/aonsectionIII_4.pdf.

[28] USAID, Demographic and health surveys methodology. 2013. Available from: http://dhsprogram.com/pubs/pdf/DHSG4/Reco de6_DHS_22March2013_DHSG4.pdf, MEASURE DHS/ICF International.

[29] Rutstein SO, Rojas G. Guide to DHS statistics. Calverton, MD: ORC Macro, 2006.

[30] Shiferaw S, Mark S, Merijn G, et al. Why do women prefer home births in Ethiopia? BMC Pregnancy and Childbirth. 2013; 13 https://doi.org/10.1186/1471-2393-13-5

[31] Federal Ministry of Health, HMIS / M\&E Indicator Definitions HMIS / M\&E Redesign: Technical Standards Area 1 version 1.0. 2007, Federal Ministry of Health: Addis Ababa, Ethiopia.

[32] Bradley S, et al. Revising unmet need for family planning. 2012 
[33] Federal Ministry of Health, HMIS Indicator Definitions: Technical Standards: Area 1, Revised Health Management Information System Indicator Definition 2014. 2014, Federal Ministry of Health: Addis Ababa, Ethiopia.

[34] Federal Ministry of Health, Monthly, quarterly and annual health service delivery reporting formats. 2014, Federal Ministry of Health,: Addis Ababa, Ethiopia.

[35] Johnson FA, Padmadas SS, Matthews Z. Are Women Deciding against Home Births in Low and Middle Income Countries? PLoS ONE. 2013; 8(6): 1-10. PMid: 23799022. https://doi.org/10 .1371 /journal pone. 0065527

[36] Mbuagbaw L, Gofin R. A New Measurement for Optimal Antenatal Care: Determinants and Outcomes in Cameroon. Maternal \& Child Health Journal. 2011; 15(8): 1427-34. PMid: 21057862. https : //doi.org/10.1007/s10995-010-0707-3

[37] MEASURE Evaluation. Family Planning and Reproductive Health Indicators Database, Percent of women receiving postpartum care by a skilled health personnel within two days of childbirth. n.d. [cited 2015 Dec 20]; Available from: http://www.cpc.unc.edu/meas ure/prh/rh_indicators/specific/sm/percent-of-women - who-received-postnatal-care-by-a.

[38] Federal Ministry of Health, National Family Planning Guideline of Ethiopia. 2011, Federal Ministry of Health: Addis Ababa, Ethiopia.

[39] Ethiopian Ministry of Finance and Economic Development (MoFED), Ethiopia Growth and Transformation Plan (GTP) 2010/11-2014/15. 2010: Addis Ababa.

[40] Federal Ministry of Health, Health Sector Development Program 1997/98- 2001/02 (EFY 1990-1994) report of the final evaluation of HSDP I 30th January to 3rd March 2003 volume 1. 2003, Federal Ministry of Health: Addis Ababa, Ethiopia.

[41] Federal Ministry of Health, Health Sector Development Programme HSDP II 2002/03 - 2004/05 (EFY 1995 - 1997), Report of the final evaluation of HSDP II 31st January - 6th March 2006 Volume I. 2006, Federal Ministry of Health: Addis Ababa, Ethiopia. 